www.jmscr.igmpublication.org

Impact Factor (SJIF): 6.379

Index Copernicus Value: 79.54

ISSN (e)-2347-176x ISSN (p) 2455-0450

crossrefDOI: https://dx.doi.org/10.18535/jmscr/v6i12.136

Journal Of Medical Science And Clinical Research

IGM Publication

An Official Publication of IGM Publication

\title{
Ultrasonographic Evidence of the Regenerative Potential of Platelet Rich Plasma In Partial Tear of Achilles Tendon - A Case Report
}

\author{
Authors \\ Arun Choudhary, Samantak Sahu², Abhimanyu Vasudeva ${ }^{3 *}$, S L Yadav \\ ${ }^{1}$ Senior Resident, Department of Physical Medicine and Rehabilitation, AIIMS, New Delhi, India \\ ${ }^{2}$ Post Graduate Trainee, Department of Physical Medicine and Rehabilitation, AIIMS, New Delhi, India \\ ${ }^{3}$ Senior Resident, Department of Physical Medicine and Rehabilitation, AIIMS, New Delhi, India \\ ${ }^{4}$ Professor, Department of Physical Medicine and Rehabilitation, AIIMS, New Delhi, India \\ *Corresponding Author
}

Abhimanyu Vasudeva

Address: A-1/124, Safdarjung Enclave, New Delhi, India 110029

Mobile: 9910185126, Email: abhimanyu.vmmc@gmail.com

\begin{abstract}
A 34 years old, female presented with severe posterior heel pain of 6 years duration. On examination, there was swelling over posterior heel near insertion of Achilles tendon with grade 3 tenderness. A point of care ultrasound examination revealed partial thickness Achilles tendon tear near its insertion as there was discontinuity of fibers, hypo-echogenicity with increased thickness. As a conservative treatment modality intra-tendinous injection of (Platelet Rich Plasma) PRP was planned. USG guided intra-tendinous injection of PRP was given thrice (4weeks apart). The patient was followed up at 3 months and 6 months after last PRP injection and had no pain or tenderness, painless and full passive and active range of motion and remarkable improvement on USG as there was restoration of fibrillar pattern, normal echogenicity and reduction in thickness. Platelet Rich Plasma (PRP) is being investigated as a safe and a minimally invasive option owing to its regenerative potential. Ultrasound is being increasingly used to visualize and diagnose disorders of the Achilles tendon.PRP is a potential regenerative therapeutic modality to restore structural and functional integrity of damaged Achillis tendon.
\end{abstract}

Keywords: PRP, Achilles Tendon, Ultrasound.

\section{Introduction}

Pain of the Achilles tendon is a common affliction in athletes as well as sedentary individuals. ${ }^{(1)}$ Tendinopathy may result from repetitive stress. Disorders of the vascular and neurological systems as well as certain rheumatologic diseases may also lead to such a condition. Achilles tendinopathy is characterized by weak and inelastic tissue and may lead to rupture as it may fail to withstand sudden stress. ${ }^{(2)} 5$ to 10 persons per 100,000 population get afflicted by Achilles tendon rupture each year and the incidence is increasing overall. ${ }^{(3)}$ Rest, immobilization and analgesics are mainstay of treatment. There is little consensus regarding conservative modalities, though Platelet Rich Plasma (PRP) is being increasingly investigated as a safe and a minimally invasive option owing to its 
regenerative potential. ${ }^{(4)}$ PRP is hypothesized to invigorate revascularization of tissues. It has also been postulated to increase the concentration of growth factors which in turn can enhance tendon healing. ${ }^{(5)}$ Ultrasound is being increasingly used to visualize and diagnose disorders of the Achilles tendon as it is an accessible, radiation free, noninvasive tool and is reliable when performed by a Physiatrist. Portability of ultrasound makes it an ideal tool for evaluation of real time sports injuries also at the time of gameplay itself and can play a pivotal role in decision making on the spot by a dedicated team of professionals led by a Physical Medicine and Rehabilitation specialist.

\section{Case Report}

A 34yearsold, female, nursing officer by profession presented in our OPD with severe posterior heel pain [Pain on Visual Analogue Scale (VAS) of 9/10] of 6 years duration. She initially received treatment from an Orthopedician, suspecting arthritis of the ankle joint and was prescribed a short course of oral steroids with minimal relief in symptoms (VAS7/10). She subsequently consulted in the Department of Rheumatology in a Government Hospital and was on regular treatment. She received Sulfasalazine on a regular basis and analgesics on as and when needed basis. However, there was no relief in symptoms (VAS-7/10). She

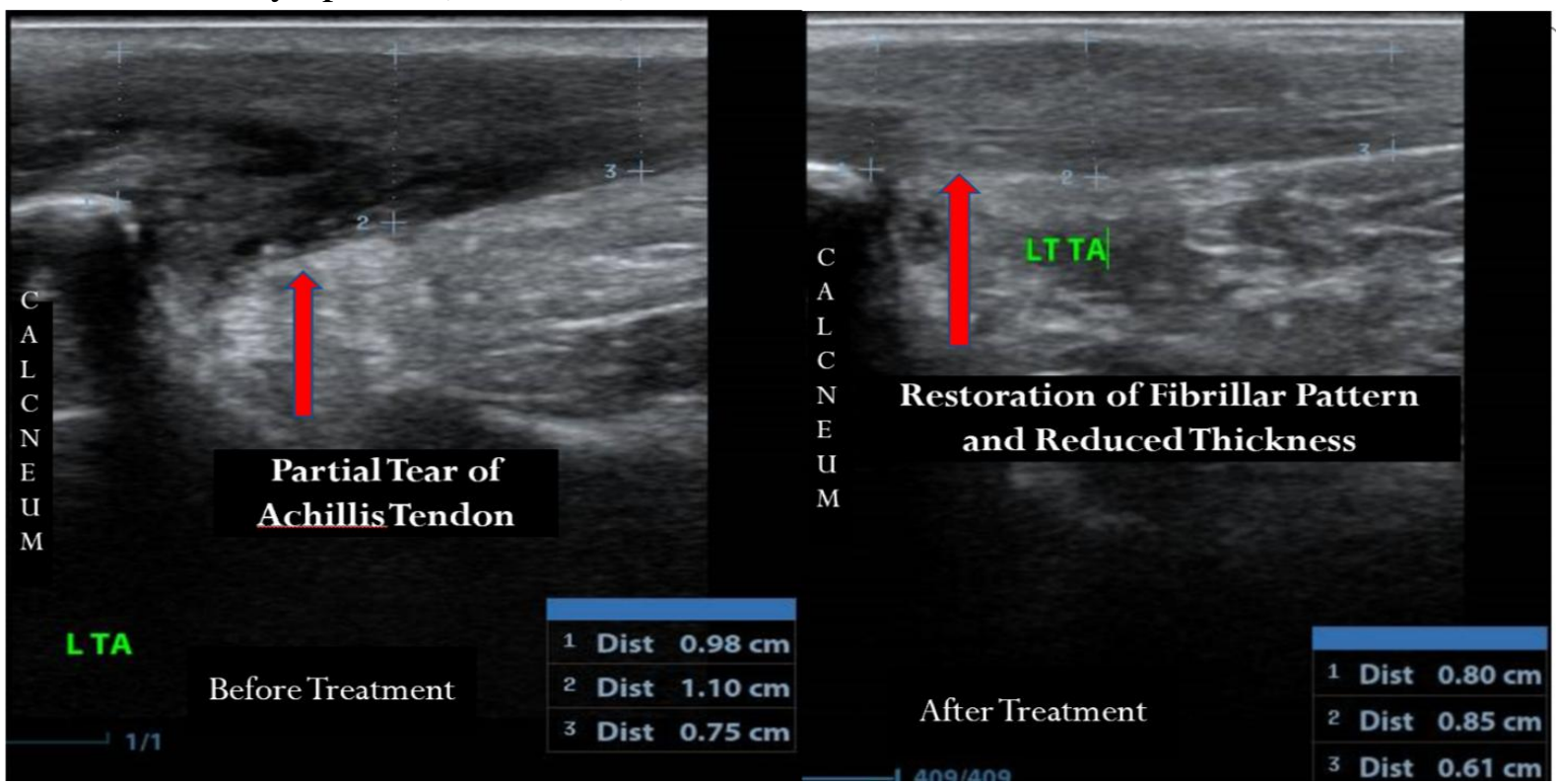

Figure-1 Ultrasonographic Structure of Achilles tendon before and after treatment also received three local injections of Methylprednisolone acetate 4 months apart. Patient had some improvement initially (VAS6/10), however, the condition deteriorated after 6 months of the last injection (VAS-9/10). The patient was referred to the Department of Physical Medicine and Rehabilitation. On examination, there was swelling over posterior heel near insertion of Achilles tendon with grade 3 tenderness. Ankle alignment and arches of foot were normal and there was no leg length discrepancy. There was no clinical evidence of retrocalcaneal bursitis. Range of motion was extremely painful. Thompson test was Negative. Footwear was also examined and it was found to be flat from the heel to the toe. The skeletal structure was normal with no joint involvement as discerned on a Radiograph of both ankle joints Antero-Posterior and Lateral views done one week earlier except for posterior calcaneal spurs on both sides. Complete Blood Count with Erythrocyte Sedimentation Rate, C-Reactive Protein, Liver and Kidney function tests were normal. Rheumatoid factor and Anti-CCP were also negative. A point of care ultrasound examination revealed partial thickness Achilles tendon tear near its insertion as there was discontinuity of fibers, hypo-echogenicity with increased thickness. 


\section{JMSCR Vol||06||Issue||12||Page 843-847||December}

36-Item Short Form Health Survey (SF-36) score, a quality of life scale with 0 being worst score and 100 being excellent, was 24 . The patient was initially treated with rest, analgesics and gentle exercises to prevent loss of motion and strength for three weeks with mild relief (VAS-7/10). As a conservative treatment modality intra-tendinous injection of PRP was planned. $2 \mathrm{ml}$ of PRP was prepared from $10 \mathrm{ml}$ of venous blood in a centrifuge machine with 1000 revolutions per minute for10minutes. USG guided intra-tendinous injection of PRP was given thrice (4weeks apart). An out of plane approach was used to minimize further injury to the tendon. A below knee cast was applied for a period of 15 days on all three occasions immediately following injection. Half an inch heel raise was advised following cast removal.

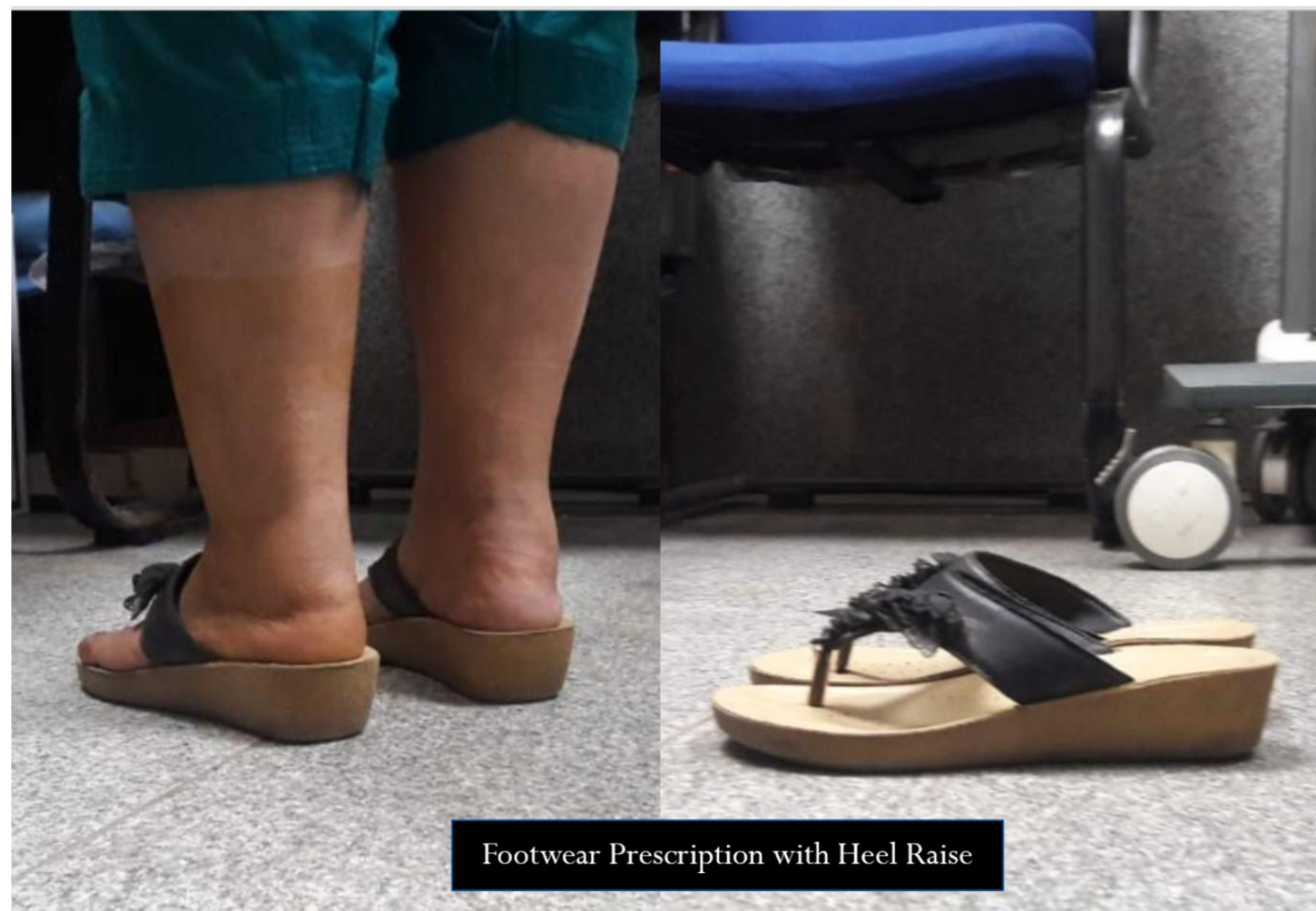

Figure-2 Footwear Prescription with Heel Raise

A home based eccentric exercise program was advised. Tablet Paracetamol and Capsule Tramodol were advised for pain relief on an SOS basis. At the end of 12 weeks, the patient reported significant relief in her symptoms. VAS was 1/10 on completion of PRP therapy and SF-36 score improved to 90 . The patient was followed up at 3 months and 6 months after last PRP injection and had no pain (VAS-0/10) or tenderness, painless and full passive and active range of motion and remarkable improvement on USG as therewas restoration of fibrillar pattern, normal echogenicity and reduction in thickness.

\section{Discussion}

Clinical diagnosis of partial tears of the Achilles tendon remains far from perfect. It is still debatable whether surgical or conservative management provides a more favorable result and, therefore, optimal treatment for such a condition remains elusive. Ultrasound is being increasingly used for diagnostic purposes. The Normal tendoachilles is of uniform thickness of $\leq 0.50 \mathrm{~cm}$ in males and $\leq 0.40 \mathrm{~cm}$ in females. In Chronic Tendinopathy, USG shows, fusiform swelling, heterogeneous echo pattern, hypoechoic foci due to mucoid degeneration and thickening of the tendon, discontinuity of the fibers, focal 
hypoechoic intra-tendinous areas, loss of fascicle organization, intra-tendinous focal calcification, partial or complete ruptures, and thickening of the hypoechoic paratenon with poorly defined borders, bursitis, and adherences between the epitenon and paratenon. The contours of the tendon may be deformed with a bumpy appearance. ${ }^{(6,7)}$ Sanches $\mathrm{M}$ et $\mathrm{al}^{(8)}$ in case-control study and descriptive laboratory study concluded that operative management of tendons combined with the application of autologous platelet rich fibrin mattress preparation rich in growth factors may present new possibilities for enhanced healing and functional recovery. Filardo $G^{(9)}$ et al treated a partial rupture of the Achilles tendon in a competitive athlete with platelet growth factors through multiple platelet-rich plasma injections with promising results. Our case also depicts excellent structural and functional recovery after PRP therapy although conflicting results exist in literature. De Vos RJ et $\mathrm{al}^{(10)}$ conducted a stratified, block-randomized, double-blind, placebo-controlled trial wherein 54 patients in the age group of 18 to 70 years with chronic tendinopathy 2 to $7 \mathrm{~cm}$ above the Achilles tendon insertion were injected either PRP or Saline(Placebo group) in addition to usual care consisting of eccentric exercises in both groups and concluded that a PRP injection compared with a saline injection did not result in a significant improvement in pain and activity.

\section{Conclusion}

PRP is a potential regenerative therapeutic modality to restore structural and functional integrity of damaged Achillis tendon. Conflicting results in literature warrant further welldesigned studies. Ultrasound is an excellent tool to diagnose and monitor such conditions in experienced hands.

\section{Sources of support: None}

\section{References}

1. Alfredson H, Lorentzon R. Chronic Achilles tendinosis: recommendations for treatment and prevention. Sports Med Auckl NZ. 2000 Feb;29(2):135-46.

2. Pedowitz D, Kirwan G. Achilles tendon ruptures. Curr Rev Musculoskelet Med. 2013 Oct 23;6(4):285-93.

3. Suchak AA, Bostick G, Reid D, Blitz S, Jomha N. The incidence of Achilles tendon ruptures in Edmonton, Canada. Foot Ankle Int. 2005 Nov;26(11):932-6.

4. Alsousou J, Keene DJ, Hulley PA, Harrison P, Wagland S, Byrne C, et al. Platelet rich Plasma in Achilles Tendon Healing 2 (PATH-

2) trial: protocol for a multicentre, participant and assessor-blinded, parallel-group randomised clinical trial comparing plateletrich plasma (PRP) injection versus placebo injection for Achilles tendon rupture. BMJ Open. 2017 Nov 1;7(11):e018135.

5. Kothari SY, Srikumar V, Singh N. Comparative Efficacy of Platelet Rich Plasma Injection, Corticosteroid Injection and Ultrasonic Therapy in the Treatment of Periarthritis Shoulder. J Clin Diagn Res JCDR. 2017 May;11(5):RC15-8.

6. Gibbon WW, Cooper JR, Radcliffe GS. Distribution of sonographically detected tendon abnormalities in patients with a clinical diagnosis of chronic achilles tendinosis. J Clin Ultrasound JCU. 2000 Feb;28(2):61-6.

7. Blankstein A. Ultrasound in the diagnosis of clinical orthopedics: The orthopedic stethoscope. World J Orthop. 2011 Feb 18;2(2):13-24.

8. Sánchez M, Anitua E, Azofra J, Andía I, Padilla S, Mujika I. Comparison of surgically repaired Achilles tendon tears using plateletrich fibrin matrices. Am J Sports Med. 2007 Feb;35(2):245-51.

9. Filardo G, Kon E, Di Matteo B, Di Martino A, Tesei G, Pelotti P, et al. Platelet-rich plasma injections for the treatment of refractory Achilles tendinopathy: results at 4 years. 
Blood Transfus Trasfus Sangue. 2014 Oct;12(4):533-40.

10. de Vos RJ, Weir A, van Schie HTM, BiermaZeinstra SMA, Verhaar JAN, Weinans H, et al. Platelet-rich plasma injection for chronic Achilles tendinopathy: a randomized controlled trial. JAMA. 2010 Jan 13;303(2):144-9. 